\title{
openheart It is time to stop counting calories, and time instead to promote dietary changes that substantially and rapidly reduce cardiovascular morbidity and mortality
}

\author{
Aseem Malhotra, ${ }^{1,2}$ James J DiNicolantonio, ${ }^{3}$ Simon Capewell ${ }^{4}$
}

To cite: Malhotra A, DiNicolantonio JJ, Capewell S. It is time to stop counting calories, and time instead to promote dietary changes that substantially and rapidly reduce cardiovascular morbidity and mortality. Open Heart 2015;2: e000273. doi:10.1136/ openhrt-2015-000273

Accepted 8 July 2015

CrossMark

1Department of Cardiology, Frimley Park Hospital, Surrey, UK

${ }^{2}$ Consultant Clinical Associate to the Academy of Medical Royal Colleges, London, UK ${ }^{3}$ Saint Luke's Mid America Heart Institute, Kansas City, Missouri, USA

${ }^{4}$ Professor of Clinical Epidemiology, University of Liverpool, Liverpool, UK

Correspondence to Dr Aseem Malhotra; aseem_malhotra@hotmail. com
Most heart attacks and ischaemic strokes are caused by complicated atheroma usually compounded by thrombosis suddenly reducing blood flow in a critical artery. Extensive evidence suggests that this atheroma silently builds up over many decades. However, arterial stiffening can be seen even in children who are obese, and aortic fatty streaks are visible in some teenagers and young adults. ${ }^{1}$ Yet, most cardiovascular events do not manifest until after the age of 60 years. The general perception is thus of a slow process that will therefore only reverse slowly, if at all. However, this perception is wrong. Extensive empirical and trial evidence reveals that substantial reductions in mortality can occur within months of quitting smoking, or making healthy dietary changes. These reductions apply to both individuals and to entire populations. In one American hospital, admissions for acute coronary syndromes decreased by $40 \%$ within 6 months of the introduction of local smoke free legislation. $^{2}$ When the law was rescinded, coronary admissions rapidly returned to previous levels. The introduction of smoke-free legislation in Scotland in 2006 was soon followed by a $6 \%$ decrease in out of hospital cardiac deaths and a $17 \%$ decrease in hospital admissions within a year. ${ }^{3}$ Even 30 min of secondhand smoke exposure has been proven to increase platelet activity and hence elevate cardiovascular risk. ${ }^{4}$

Similarly, changes in diet can rapidly improve outcomes of cardiovascular disease (CVD), as demonstrated by several randomised trials. In the DART trial, 2033 survivors of myocardial infarction who were advised to eat fatty fish had a significant $29 \%$ reduction in all-cause mortality compared with control patients, with survival curves separating within months. Likewise, in the Gruppo Italiano per lo Studio della
Sopravvivenza nell'Infarcto Miocardico (GISSI)-Prevention trial, $1 \mathrm{~g}$ of $\Omega-3$ fatty acids significantly reduced all-cause mortality and cardiovascular mortality in 11324 myocardial infarction survivors. Moreover, survival curves separated early, with a significant reduction in total mortality after just 3 months of treatment $(\mathrm{p}=0.037) .^{5}$

The PREvencion con DIeta MEDiterranea (PREDIMED) primary prevention randomised controlled trial found that an energy unrestricted diet supplemented with extra virgin olive oil or nuts achieved an impressive $30 \%$ reduction in major cardiovascular events $(\mathrm{NNT}=61)$ in over 7500 high risk individuals initially free of CVD. This reduction occurred within 3 months. ${ }^{6}$ Furthermore, this solid RCT evidence builds on a wealth of existing data from observational, cohort and secondary prevention intervention studies. ${ }^{7}{ }^{8}$ It also provides further strong causal evidence that simple diet interventions can rapidly and powerfully reduce CVD outcomes. In comparison with an American Heart Association recommended 'low fat' diet, a Mediterranean diet post myocardial infarction is a more powerful coronary intervention tool for mortality than aspirin, statins, or coronary stents, but without any significant difference in total cholesterol, triglycerides or HDL between the two groups. ${ }^{9}$ It is the abundant $\alpha$-linoleic acid, polyphenols and $\Omega-3$ fatty acids found in nuts, olive oil, oily fish and vegetables, that rapidly exert positive health effects by attenuating inflammation, atherosclerosis and thrombosis. ${ }^{10}$ Conversely, the consumption of transfats commonly found in fast food can rapidly increase $\mathrm{C}$ reactive protein and other inflammatory markers within weeks. ${ }^{11}$

Strategies that prevent excessive weight gain in children and adults through curbing the consumption of the amounts of unhealthful foods should also be welcomed. However, 
simply focusing on weight loss in obese subjects misses a key finding from analysis of PREDIMED subgroups: dietary intervention achieved consistently large reductions in CVD risk irrespective of weight. Furthermore, weight loss interventions are rarely sustained. The weight loss industry, which emphasises calorie restriction over good nutrition, generates $\$ 58$ billion in revenue annually in the USA, even though long-term follow-up studies reveal that the majority of individuals regain virtually all of the weight that was lost during treatment irrespective of whether they maintain their diet or exercise programme. ${ }^{12}$ Shifting focus away from calories and emphasising a dietary pattern that focuses on food quality rather than quantity will help to rapidly reduce obesity, related diseases and cardiovascular risk. ${ }^{13}{ }^{14}$ Rapid weight loss and regain that can occur from fad dieting is actually detrimental to health. Such 'weight cycling' contributes to hypertension, insulin resistance and dyslipidaemia resulting in increased mortality risk and worse cardiovascular outcomes. ${ }^{15}$ The look AHEAD (Action for Health in Diabetes) trial found no reduction in the composite endpoint (ie, death from cardiovascular causes, non-fatal myocardial infarction, non-fatal stroke, or hospitalisation for angina) with a low calorie diet (on top of increased physical activity) in patients with type 2 diabetes despite a maximum follow-up of 13.5 years and despite significant weight loss in the intervention group. ${ }^{16}$

In a randomised, controlled, double-blinded dietary intervention trial for blood pressure, compared to placebo, the daily ingestion of flaxseed (high in $\Omega-3$, lignans and fibre) induced a significant $10 \mathrm{~mm} \mathrm{Hg}$ systolic $/ 7 \mathrm{~mm} \mathrm{Hg}$ diastolic blood pressure reduction in hypertensive patients with peripheral arterial disease, within 6 months. The rate of stroke and myocardial infarction was cut in half in the flaxseed group compared to the placebo group. Furthermore, there was no significant difference in weight change between the two groups. ${ }^{17}$

The American Heart Association predicts that 8 million Americans will have heart failure by 2030. The total direct costs will triple from approximately $\$ 20$ billion in 2012 to $\$ 70$ billion in $2030 .{ }^{18}$ Recently, a small study suggested that a low-carbohydrate diet in patients with diabetes reversed echocardiographic markers of diastolic dysfunction within weeks of implementation. ${ }^{19}$ This finding is all the more valuable considering there is, to date, no proven pharmacological intervention that improves prognosis in diastolic heart failure, which affects $30-50 \%$ of all heart failure patients. ${ }^{19}$

An exaggerated belief in the (modest) benefits of pharmacotherapy, ${ }^{20}$ aggressively reinforced by commercial vested interests, can often mislead patients and doctors, and promotes overtreatment in chronic disease management, and may even distract from and undermine the benefits of simple lifestyle interventions.

Focusing on total energy consumed, as opposed to nutritional value, has been exploited by the food industry, which has added sugar to over $80 \%$ of all processed foods. One can of cola contains nine teaspoons of sugar.
The EPIC study revealed that one can a day (approximately 150 calories) was associated with substantially increasing the risk of developing type 2 diabetes. ${ }^{21}$ Conversely, PREDIMED revealed that consumption of a handful of nuts, $(30 \mathrm{~g}$ of walnuts, $15 \mathrm{~g}$ of almonds and $15 \mathrm{~g}$ of hazelnuts) or four tablespoons of extra virgin olive oil per day (approximately 500 calories) significantly reduced the risk of heart attack and stroke. A recent randomised pilot study of a calorie unrestricted very low carbohydrate/high fat diet in overweight patients with type 2 diabetes or prediabetes resulted in a significant improvement in glycaemic control and even discontinuation of diabetes medications within 3 months in comparison to a moderate carbohydrate, low-fat calorie-restricted diet (consistent with guidelines from the American Diabetes Association), with no adverse effect on blood lipids. ${ }^{22} \mathrm{~A}$ critical review in Nutrition also concluded that dietary carbohydrate restriction is the "single most effective intervention for reducing all of the features of the metabolic syndrome" and should be the first approach in diabetes management with the very low carbohydrate ketogenic diet $(<10 \%$ carbs $)$ revealing the greatest falls in glycated hemoglobin and reduction in the use of medications with benefits occurring even without weight loss. ${ }^{23}$

Primary and secondary care clinicians clearly have a duty to their individual patients and also to their local populations. Our continued collective failure to act is an option we cannot afford. Obesity alone is already costing the National Health Service (NHS) over $£ 5$ billion a year. Total costs of type 2 diabetes in the UK exceed $£ 20$ billion and are predicted to double in the next 20 years. ${ }^{24}$ Similarly, in the USA, the cost of diabetes has risen $40 \%$ in the past 5 years, reaching $\$ 245$ billion in 2012. ${ }^{25}$ The extensive Global Burden of Disease studies show that poor diet is consistently responsible for more disease and death than physical inactivity, smoking and alcohol combined. ${ }^{26}$ This global disease burden will clearly not be prevented by medications; it will require policy interventions that make healthier diet choices easier (the 'default option'). ${ }^{27}$ The most powerful and effective policies include taxation on sugary drinks, and subsidies to increase the affordability and availability of healthier foods including nuts, vegetables and fruit, in addition to controls on the marketing of junk foods and clear package labelling. Increasing nut consumption among the American population by two servings per week could prevent 90000 CVD deaths per year. ${ }^{28}$ Applying these population-wide policies might achieve rapid reductions in disease and hospital admissions visible even within the electoral term of most politicians. It is time to stop counting calories, and time to instead promote good nutrition and dietary changes that can rapidly and substantially reduce cardiovascular mortality. The evidence indeed supports the mantra that "food can be the most powerful form of medicine or the slowest form of poison". Recommending a high fat Mediterranean-type diet and lifestyle to our patients, friends and families, might be a good place to start. 
Twitter Follow Aseem Malhotra at @DrAseemMalhotra

Contributors AM and SC wrote the initial draft and JJD helped revise the arguments, wrote parts of the paper and included several additional references.

Funding JJD works for a company that sells nutriceuticals, but he is not involved in the selling or marketing of these products. SC is a non-trustee for the UK Faculty of Public Health, The UK Health Forum and Heart of Mersey.

Competing interests None declared.

Provenance and peer review Commissioned; internally peer reviewed.

Open Access This is an Open Access article distributed in accordance with the Creative Commons Attribution Non Commercial (CC BY-NC 4.0) license, which permits others to distribute, remix, adapt, build upon this work noncommercially, and license their derivative works on different terms, provided the original work is properly cited and the use is non-commercial. See: http:// creativecommons.org/licenses/by-nc/4.0/

\section{REFERENCES}

1. Strong JP, Malcom GT, McMahan CA, et al. Prevalence and extent of atherosclerosis in adolescents and young adults: implications for prevention from the Pathobiological Determinants of Atherosclerosis In Youth Study. JAMA 1999;281:727-35.

2. Sergent RP, Shephard RM, Glantz SA. Reduced incidence of admissions for myocardial infarctions associated with public smoking ban. Before and after study. BMJ 2004;328:977-80.

3. Meyers DG, Neuberger JS, He J. Cardiovascular eff ect of bans on smoking in public places: a systematic review and meta-analysis. J Am Coll Cardiol 2009;54:1249-55.

4. Barnoya B, Stanton A. Cardiovascular effects of second hand smoke. Circulation 2005;111:2684-98.

5. [No authors listed]. Dietary supplementation with n-3 polyunsaturated fatty acids and vitamin $E$ after myocardial infarction: results of the GISSI-Prevenzione trial. Gruppo Italiano per lo Studio della Sopravvivenza nell'Infarto miocardico. Lancet 1999;354:447-55.

6. Estruch R, Ros E, Salas-Salvadó J, et al, PREDIMED Study Investigators. Primary prevention of cardiovascular disease with a Mediterranean diet. N Engl J Med 2013;368:1279-90.

7. De Lorgeril M, Salen P, Martin JL, et al. Mediterranean diet, traditional risk factors, and the rate of cardiovascular complications after myocardial infarction: final report of the Lyon Diet Heart Study. Circulation 1999;99:779-85.

8. Capewell S, O'Flaherty M. Can dietary changes rapidly decrease cardiovascular mortality rates? Eur Heart J 2011;32:1187-9.

9. Quaas J. Mediterranean diet for secondary prevention after heart attack. The NNT, 17 Sept 2010. http://www.thennt.com/nnt/ mediterranean-diet-for-post-heart-attack-care/

10. Chakrabarti S, Freedman JE. Review: nutriceuticals as antithrombotic agents. Cardiovasc Ther 2010;28:227-35.

11. Wallace $S$, Mozaffarian D. Trans-fatty acids and non lipid risk factors. Curr Atheroscler Rep 2009;11:423-33.
12. Strohacker K, McFarlin B. Influence of obesity, physical inactivity, and weight cycling on chronic inflammation. Front Biosci 2010; E2:98-104.

13. Montani JP, Viecelli AK, Prevot A, et al. Weight cycling during growth and beyond as a risk factor for later cardiovascular diseases: the'repeated overshoot' theory. Int J Obes (Lond) 2006;30(Suppl 4): S58-66.

14. Lucan SC, DiNicolantonio JJ. How calorie-focused thinking about obesity and related diseases may mislead and harm public health. An alternative. Public Health Nutr 2015;18:571-81.

15. Mann T, Tomiyama AJ, Westling E, et al. Medicare's search for effective obesity treatments: diets are not the answer. Am Psychol 2007;62:220-33.

16. Wing RR, Bolin P, Brancati FL, et al. Look AHEAD Research Group. Cardiovascular effects of intensive lifestyle intervention in type 2 diabetes. N Engl J Med 2013;369:145-54.

17. Rodriguez-Leyva D, Weighell W, Edel AL, et al. Potent antihypertensive action of dietary flaxseed in hypertensive patients. Hypertension 2013;62:1081-9.

18. Heidenreich PA, Albert NM, Allen LA, et al. Council on Epidemiology and Prevention; Stroke Council. Forecasting the impact of heart failure in the United States: a policy statement from the American Heart Association. Circ Heart Fail 2013;6:606-19.

19. von Bibra $\mathrm{H}$, Wulf $\mathrm{G}$, Pfützner $\mathrm{A}$, et al. A low glycemic/insulinemic diet improves diastolic cardiac function and metabolic syndrome more than the traditional low-fat diet in overweight patients with type 2 diabetes. Prediabetes and the Metabolic Syndrome 2013 Congress; 19 April 2013, Vienna, Austria. Abstract 852.

20. Diamond DM, Ravnskov U. How statistical deception created the appearance that statins are safe and effective in primary and secondary prevention of cardiovascular disease. Expert Rev Clin Pharmacol 2015;8:201-10.

21. Romaguera D, Norat T, Wark PA., InterAct Consortium. Consumption of sweet beverages and type 2 diabetes incidence in European adults: results from EPIC-InterAct. Diabetologia 2013;56:1520-30.

22. Saslow LR, Kim S, Daubenmier JJ, et al. A randomized pilot trial of a moderate carbohydrate diet compared to a very low carbohydrate diet in overweight or obese individuals with type 2 diabetes mellitus or prediabetes. PLoS ONE 2014;9:e91027.

23. Feinman RD, Pogozelski WK, Astrup A, et al. Dietary carbohydrate restriction as the first approach in diabetes management: critical review and evidence base. Nutrition 2015;31:1-13.

24. Hex N, Bartlett $C$, Wright D, et al. Estimating the current and future costs of type 1 and type 2 diabetes in the United Kingdom, including direct health costs and indirect societal and productivity costs. Diabet Med 2012;29:855-62.

25. Tucker ME. Diabetes cost the US $\$ 245$ billion in 2012. Web MD, 8 March 2013. http://diabetes.webmd.com/news/20130308/ diabetes-costs-rising

26. Global Burden of Diseases, Injuries, and Risk Factors Study 2013. The Lancet, 22 Jul 2014. http://thelancet.com/global-burden-ofdisease

27. Frieden TR. A framework for public health action: the health impact pyramid. Am J Public Health 2010;100:590-5.

28. Mozaffarian D, Capewell S. United Nations' dietary policies to prevent cardiovascular disease. BMJ 2011;343:d5747. 